Full text at publisher Full Text Links
Export $\vee$

Add to Marked List

\title{
Biotransformation of sesquiterpenoids: a recent insight
}

By: Aminudin, NI (Aminudin, Nurul Iman) ${ }^{1}$; Ridzuan, M (Ridzuan, Munirah) ${ }^{1}$; Susanti, D (Susanti, Deny) ${ }^{1}{ }^{\text {; Azira, }}$ Z (Azira, Zaima) ${ }^{2}$; Abidin, Z (Abidin, Zainal) ${ }^{2}$ View Web of Science ResearcherID and ORCID (provided by Clarivate)

JOURNAL OF ASIAN NATURAL PRODUCTS RESEARCH

DOI: $10.1080 / 10286020.2021 .1906657$

Early Access: MAR 2021

Document Type: Article; Early Access

\section{Abstract}

Sesquiterpenoids have been identified as natural compounds showing remarkable biological activities found in medicinal plants. There is great interest in developing methods to obtain sesquiterpenoids derivatives and biotransformation is one of the alternative methods for structural modification of complex sesquiterpenes structures. Biotransformation is a great drug design tool offering high selectivity and green method. The present review describes a comprehensive summary of biotransformation products of sesquiterpenoids and its structural modification utilizing a variety of biocatalysts including microorganisms, plant tissue culture and enzymes. This review covers recent literatures from 2007 until 2020 and highlights the experimental conditions for each biotransformation process.

\section{Keywords}

Author Keywords: Biotransformation; sesquiterpenoids; biocatalysts; drug-design

\section{Author Information}

Corresponding Address: Aminudin, Nurul Iman (corresponding author)

- Int Islamic Univ Malaysia IIUM, Kulliyyah Sci, Dept Chem, Kuantan 25200, Pahang, Malaysia Addresses:

- ${ }^{1}$ Int Islamic Univ Malaysia IIUM, Kulliyyah Sci, Dept Chem, Kuantan 25200, Pahang, Malaysia

- 2 Int Islamic Univ Malaysia IIUM, Kulliyyah Sci, Dept Biotechnol, Kuantan 25200, Pahang, Malaysia

E-mail Addresses: nuruliman@iium.edu.my

\section{Categories/Classification}

Research Areas: Plant Sciences; Chemistry; Pharmacology \& Pharmacy

\section{Funding}

\section{Funding agency}

Ministry of Higher Education (MOHE) Malaysia through Fundamental Grant Scheme

\section{Grant number}

FRGS19-029-0637

FRGS/1/2018/STG01/UIAM/03/3

View funding text

\section{+ See more data fields}

\section{Journal information}

Journal Of Asian Natural Products Research

ISSN: $1028-6020$

eISSN: $1477-2213$

Current Publisher: TAYLOR \& FRANCIS LTD, 2-4 PARK SQUARE, MILTON PARK,

ABINGDON OR14 4RN, OXON, ENGLAND

Journal Impact Factor: Journal Citation Report ${ }^{\text {TM }}$

Research Areas: Plant Sciences; Chemistry; Pharmacology \& Pharmacy

Web of Science Categories: Plant Sciences; Chemistry, Applied; Chemistry, Medicinal; Pharmacology \& Pharmacy

\section{Citation Network}

In Web of Science Core Collection

0

Citations

Create citation alert

Cited References

\section{9}

View Related Records

You may also like...

COLLINS, M;

PLANT-TISSUE CULTURE IN NON-LABORATORY

CONDITION

OHIO JOURNAL OF SCIENCE

Chellan, S; Al-Shayji, Y; Al-Ajeel, A; et al. Salt-Tolerant Potato Developed via Plant Tissue Culture

IN VITRO CELLULAR \& DEVELOPMENTAL BIOLOGY-ANIMAL

WULLEMS, GJ;

PLANT-TISSUE CULTURE AND ITS BIOTECHNOLOGICAL APPLICATION MONATSSCHRIFT FUR BRAUWISSENSCHAFT

Martin-Closas, LL; Mateo, L; Pelacho, AM; Plant tissue culture as a tool to reveal Papaver rhoeas resistant biotypes to tribenuron methyl COMPARATIVE BIOCHEMISTRY AND PHYSIOLOGY A-MOLECULAR \& INTEGRATIVE PHYSIOLOGY

Sharma, DP;

Using Plant Tissue Culture to Generate MosaicFree Fig Plants and Select Salt Tolerant Pistachio Rootstocks IN VITRO CELLULAR \& DEVELOPMENTAL BIOLOGY-ANIMAL

See all

\section{Use in Web of Science}

Web of Science Usage Count

1

Last 180 Days

Factor $^{\mathrm{TM}}$

(2019)

Learn more 
79 Cited References

Showing 30 of 79

View as set of results

(from Web of Science Core Collection) 

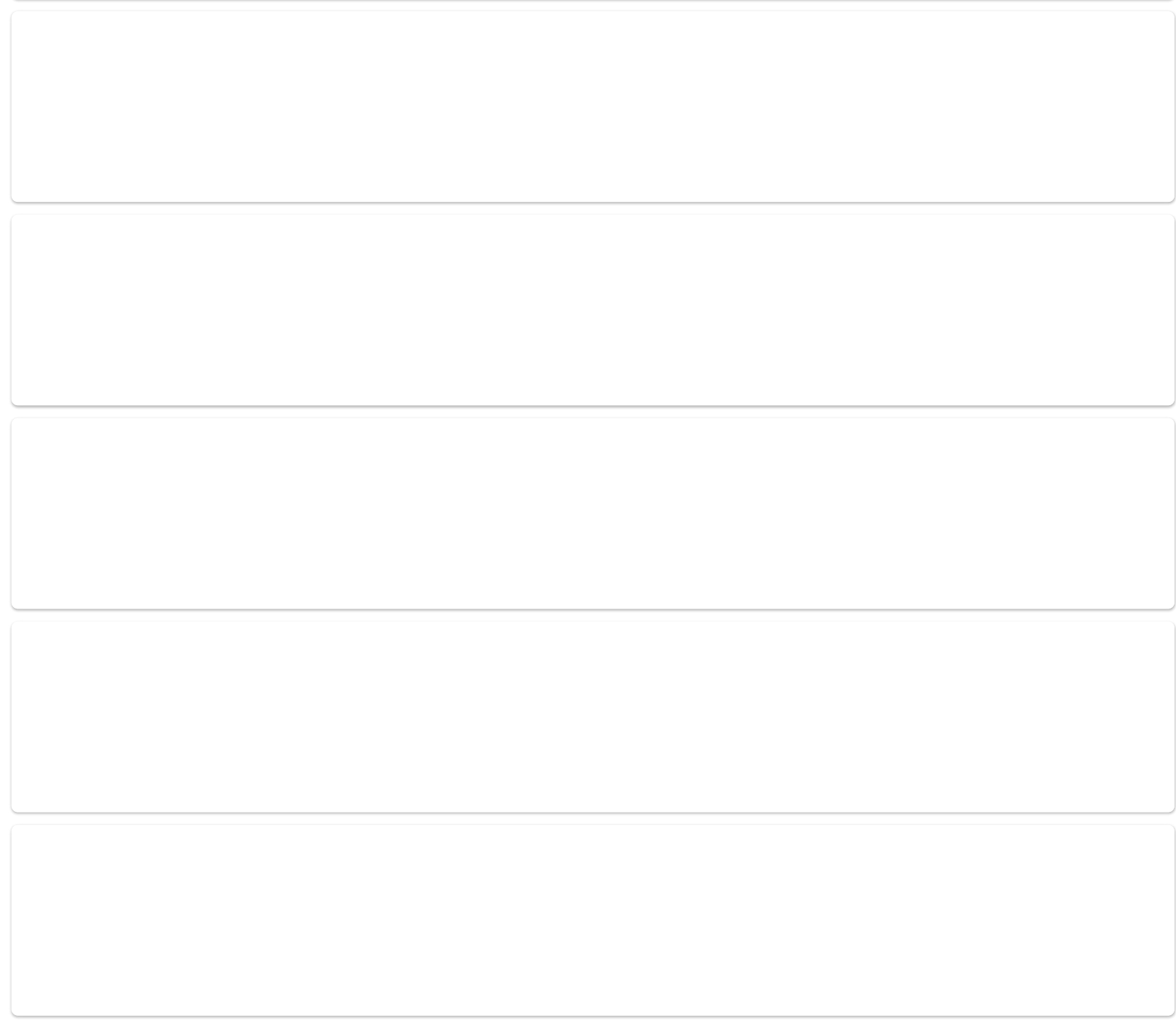


$\begin{array}{llll}\text { (c) 2021 Clarivate } & \text { Data Correction } & \text { Copyright Notice } & \text { Follow Us } \\ \text { Training Portal } & \text { Privacy Statement } & \text { Cookie Policy } & \text { y } \\ \text { Product Support } & \text { Newsletter } & \text { Terms of Use } & \end{array}$

